\title{
Axial Dissipative Dust as a Source of Gravitational Radiation in $f(R)$ Gravity
}

\author{
M. Sharif *and Aisha Siddiqa ${ }^{\dagger}$ \\ Department of Mathematics, University of the Punjab, \\ Quaid-e-Azam Campus, Lahore-54590, Pakistan.
}

\begin{abstract}
In this paper, we explore the source of gravitational radiation in the context of $f(R)$ gravity by considering axially symmetric dissipative dust under geodesic condition. We evaluate scalars associated with electric and magnetic parts of the Weyl tensor for both non-spinning (at the center) and spinning (in the surrounding of the center) fluids of the configuration. For this purpose, we use the evolution as well as constraint equations for kinematical quantities and Weyl tensor. Finally, we investigate the existence of gravitational radiation through super-Poynting vector. It is found that the fluid is not gravitationally radiating in the non-spinning case but it is gravitationally radiating for the spinning case.
\end{abstract}

Keywords: $f(R)$ gravity, Axial source, Gravitational radiation.

PACS: 04.50.Kd; 04.40.Nr; 04.40.Dg.

\section{Introduction}

Gravitational waves (GWs) are ripples in the curvature of spacetime produced by moving objects and gravitational radiation is the energy carried by

*msharif.math@pu.edu.pk

†aisha.siddiqa17@yahoo.com 
these waves. However, gravity is the weakest of the fundamental forces so these waves are not detectable even through sensitive detectors unless produced by the massive stellar objects. One of the important features of GWs is that if we could detect and observe these waves then it will lead new observational techniques for different astrophysical phenomena. Recently, LIGO scientific collaboration and Virgo collaboration [1] provided the first observational evidence for GWs originating from a pair of merging black holes. Hawking [2] showed that there is an upper bound for the energy of gravitational radiation emitted by the collision of two black holes. Wagoner [3] investigated the gravitational radiation produced by accreting neutron stars. Flanagan and Hughes [4] provided a comprehensive study of many important aspects about theory of GWs. They found that linearized theory is appropriate to explain GWs propagation, interaction of these waves with detectors and emission of GWs from a source. They also discussed different formalisms to deal with the situations where linearized treatment is not sufficient.

Dust solutions are considered to be ideal models for astrophysical objects in which particles are assumed to interact only gravitationally. Ellis [5] investigated dynamics of dust fluid and found some new solutions of the field equations. Lemos [6] showed that naked singular solutions formed by gravitational collapse of radiation and dust are same in nature. Dissipation is an important phenomenon in the evolution of stellar objects. It is, in fact, the only mechanism that leads to a star away from hydrostatic equilibrium by neutrino emission. Although, pressure is present in a physical process with dissipative fluid, however, some authors neglect it to see the effects of dissipation on the system as well as for analytical solution. The dissipative dust cloud has been considered by many researchers [7] to investigate the causes of energy density inhomogeneity in GR as well as modified gravity theories. Herrera et al. 88 discussed the existence of gravitational radiation in dissipative dust. Thus it would be worthwhile to study the evolution of celestial bodies through dissipative dust.

It is well-known that astrophysical systems can be rotating and hence their exterior solutions cannot be exactly spherically symmetric. The region outside a rotating stellar body can be represented only by an axial symmetric spacetime, the most compatible for the interior region [9]. According to Bhirkoff theorem, spherically symmetric spacetime is not appropriate while axially symmetric spacetime is compatible with the existence of gravitational radiation [10. Herrera et al. [8, 11] investigated the presence of gravitational radiation in general relativity (GR) for perfect and dissipative dust fluid with 
axial symmetry under the geodesic condition. They showed that both types of fluid do not produce gravitational radiation.

The Weyl tensor represents that part of curvature which cannot be determined by matter locally. However, the contracted Bianchi identities serve as field equations for the Weyl tensor and give the part of curvature depending on matter 9]. This approach is used to discuss gravitational radiation in the literature [12. Matte [13] proposed electric and magnetic parts of the Weyl tensor to show an analogy between gravitational waves and electromagnetic waves. Bertschinger and Hamilton [14] derived the evolution equations for these parts of the Weyl tensor. Maartens and Basset [15] introduced superPoynting vector to describe the flux of super energy which depends upon the magnetic and electric parts of the Weyl tensor. When super-Poynting vector is non-zero it is defined to be a state of intrinsic gravitational radiation [16.

The mystery of dark energy leads to the concept of modified theories of gravity. $f(R)$ gravity is one of the viable modified theories in which Ricci scalar in the Einstein-Hilbert action is replaced by its generic function $f(R)$. Starobinsky [17] proposed the first inflationary model in $f(R)$ which is compatible with anisotropies of CMBR (Cosmic Microwave Background Radiation). Bamba et al. [18] introduced $f(R)$ model which explains inflation and late cosmic expansion at the same time. There are also other models in $f(R)$ gravity [19, 20] that deal with cosmological constraints and can resolve some cosmic issues.

Cembranos et al. 21] investigated spherical dust collapse in this gravity and showed that collapsing process slows down due to the contribution of $f(R)$ terms. Sharif and Yousaf [22] discussed the collapse with metric and Palatini $f(R)$ gravity considering early and late time models. Sharif and $\mathrm{Zu}-$ naira [23, 24] explored the effects of this gravity on axially symmetric fluid under shear-free and expansion-free conditions with the help of structure scalars. Capozziello et al. [25] investigated axially symmetric solutions in this gravity using Noether symmetry approach and discussed physical properties of the fluid. Rippl et al. [26] studied the evolution equations for kinematical variables, electric and magnetic parts of the Weyl tensor. Näf and Jetzer [27] described gravitational radiation of an isolated system in $f(R)$ quadratic model. They used correspondence between $f(R)$ as well as scalartensor theories and found results inconsistent with GR. The phenomenon of gravitational waves has also been studied in this gravity [28].

In order to analyze the influence of higher order curvature terms $(f(R)$ gravity) upon the phenomenon of gravitational radiation, we explore the 
evolution of dissipative dust configuration in $f(R)$ gravity. The paper has following format. In the coming section, a basic formalism of $f(R)$ gravity and the necessary ingredients to discuss evolution of the fluid are given. Sections 3 and 4 deal with evolution of non-spinning and spinning fluids, respectively. The last section provides summary of the results obtained.

\section{Basic Formalism}

The action of $f(R)$ gravity is defined as

$$
S=\frac{1}{16 \pi} \int \sqrt{-g} f(R) d^{4} x+S_{M},
$$

where $S_{M}=\int \sqrt{-g} L_{M} d^{4} x$ shows the matter action in which $L_{M}$ represents the matter Lagrangian. The field equations obtained by varying Eq.(27) with respect to the metric tensor are given by

$$
R_{\beta \gamma}-\frac{1}{2} R g_{\beta \gamma}=8 \pi\left\{T_{\beta \gamma}^{(m)}+\frac{1}{8 \pi} T_{\beta \gamma}^{(D)}\right\}=8 \pi T_{\beta \gamma}^{\text {tot }} .
$$

Here $T_{\beta \gamma}^{(m)}$ represents the energy-momentum tensor of matter and $T_{\beta \gamma}^{(D)}$ shows the contribution of dark source terms defined as

$$
T_{\beta \gamma}^{(D)}=\frac{1}{8 \pi}\left\{(1-F) R_{\beta \gamma}+\frac{g_{\beta \gamma}}{2}(f-R)+\nabla_{\beta} \nabla_{\gamma} F-g_{\beta \gamma} \square F\right\},
$$

where $F=\frac{d f}{d R}$ and $\square=\nabla^{\nu} \nabla_{\nu}$. The density $\left(\rho^{(D)}\right)$, pressure $\left(p^{(D)}\right)$ and energy flux $\left(q_{\alpha}^{(D)}\right)$ associated with the dark source are given by [29]

$$
\rho^{(D)}=T_{\beta \gamma}^{(D)} V^{\beta} V^{\gamma}, \quad p^{(D)}=\frac{1}{3} T_{\beta \gamma}^{(D)} h^{\beta \gamma}, \quad q_{\alpha}^{(D)}=-T_{\beta \gamma}^{(D)} V^{\beta} h_{\alpha}^{\gamma},
$$

where $V^{\beta}$ is the four velocity and $h^{\beta \gamma}$ denotes the projection tensor. The axially symmetric spacetime with reflection symmetry and geodesic condition is given by [11]

$$
d s^{2}=-d t^{2}+B^{2}(t, r, \theta)\left(d r^{2}+r^{2} d \theta^{2}\right)+2 \tilde{G}(r, \theta) d t d \theta+C^{2}(t, r, \theta) d \phi^{2} .
$$

The energy-momentum tensor of dust suffering heat dissipation in the form of radiation is given by

$$
T_{\beta \gamma}^{(m)}=\rho V_{\beta} V_{\gamma}+q_{\beta} V_{\gamma}+q_{\gamma} V_{\beta},
$$


where $\rho$ is the energy density, $q_{\gamma}$ indicates the heat flux vector and $V_{\gamma}$ shows the four velocity. The four velocity and unit spacelike vectors in comoving coordinates are defined as

$$
\begin{aligned}
& V_{\gamma}=(-1,0, \tilde{G}, 0), \quad K_{\gamma}=(0, B, 0,0), \\
& L_{\gamma}=\left(0,0, \sqrt{r^{2} B^{2}+\tilde{G}^{2}}, 0\right), \quad S_{\gamma}=(0,0,0, C) .
\end{aligned}
$$

These vectors describe a set of orthonormal tetrad and satisfy the following relations

$$
\begin{aligned}
V_{\gamma} V^{\gamma} & =-K^{\gamma} K_{\gamma}=-L^{\gamma} L_{\gamma}=-S^{\gamma} S_{\gamma}=-1, \\
V_{\gamma} K^{\gamma} & =V^{\gamma} L_{\gamma}=V^{\gamma} S_{\gamma}=K^{\gamma} L_{\gamma}=K^{\gamma} S_{\gamma}=S^{\gamma} L_{\gamma}=0 .
\end{aligned}
$$

Since, $G_{03}$ is zero, so from the field equations, we have $T_{03}^{\text {tot }}=0$ which implies that $q_{3}^{\text {tot }}=0$. Thus we can write

$$
q_{\alpha}^{t o t}=q_{I}^{t o t} K_{\alpha}+q_{I I}^{t o t} L_{\alpha}
$$

Also, we have

$$
q_{\alpha}^{t o t}=-T_{\beta \gamma} V^{\beta} V^{\gamma} V_{\alpha}-T_{\alpha \beta} V^{\beta}
$$

These two equations yield

$$
\begin{aligned}
q_{I}^{\text {tot }} & =-\frac{1}{B} T_{01}^{\text {tot }} V^{0}=q_{I}-\frac{1}{8 \pi B} T_{01}^{(D)}, \\
q_{I I}^{t o t} & =-\frac{1}{\sqrt{r^{2} B^{2}+\tilde{G}^{2}}}\left\{\tilde{G} T_{00}^{(D)}+\sqrt{r^{2} B^{2}+\tilde{G}^{2}} q_{I I}+T_{02}^{(D)}\right\} .
\end{aligned}
$$

Now we define kinematical quantities as well as electric and magnetic parts of the Weyl tensor. The kinematical variables are very important tools to discuss the evolution of any astrophysical system. These variables include four acceleration, expansion scalar, shear tensor, and vorticity tensor. Since we are considering geodesic fluid, so the four acceleration is zero. The expansion scalar is given by

$$
\Theta=V_{; \gamma}^{\gamma}=\left(\frac{2 r^{2} B^{2}+\tilde{G}^{2}}{r^{2} B^{2}+\tilde{G}^{2}}\right) \frac{\dot{B}}{B}+\frac{\dot{C}}{C},
$$

where dot represents derivative with respect to $t$. The shear tensor is defined as

$$
\sigma_{\beta \gamma}=V_{(\beta ; \gamma)}+a_{(\beta} V_{\gamma)}-\frac{1}{3} \Theta h_{\beta \gamma}
$$


whose non-zero components are $\sigma_{11}, \sigma_{22}$ and $\sigma_{33}$. In terms of unit spacelike vectors, we can have

$$
\sigma_{\beta \gamma}=\frac{1}{3}\left(2 \sigma_{I}+\sigma_{I I}\right)\left(K_{\beta} K_{\gamma}-\frac{1}{3} h_{\beta \gamma}\right)+\frac{1}{3}\left(2 \sigma_{I I}+\sigma_{I}\right)\left(L_{\beta} L_{\gamma}-\frac{1}{3} h_{\beta \gamma}\right),
$$

where

$$
\sigma_{I}=\left(\frac{r^{2} B^{2}+2 \tilde{G}^{2}}{r^{2} B^{2}+\tilde{G}^{2}}\right) \frac{\dot{B}}{B}-\frac{\dot{C}}{C}, \quad \sigma_{I I}=\left(\frac{r^{2} B^{2}-\tilde{G}^{2}}{r^{2} B^{2}+\tilde{G}^{2}}\right) \frac{\dot{B}}{B}-\frac{\dot{C}}{C},
$$

giving

$$
\sigma_{I}-\sigma_{I I}=\frac{3 \tilde{G}^{2}}{r^{2} B^{2}+\tilde{G}^{2}} \frac{\dot{B}}{B}
$$

The vorticity tensor is

$$
\Omega_{\beta \gamma}=V_{[\beta ; \gamma]}+a_{[\beta} V_{\gamma]},
$$

whose non-zero component is $\Omega_{12}=\frac{-\tilde{G}^{\prime}}{2}$, implying that

$$
\Omega_{\beta \gamma}=\Omega\left(L_{\beta} K_{\gamma}-L_{\gamma} K_{\beta}\right) \text {, }
$$

where $\Omega$ is the vorticity scalar given by

$$
\Omega=\Omega^{\beta \gamma} \Omega_{\beta \gamma}=\frac{\tilde{G}^{\prime}}{2 B \sqrt{r^{2} B^{2}+\tilde{G}^{2}}} .
$$

Here prime denotes differentiation with respect to the radial coordinate $r$.

The electric and magnetic parts of the Weyl tensor are defined as

$$
E_{\beta \gamma}=C_{\beta \delta \gamma \lambda} V^{\delta} V^{\lambda}, \quad H_{\beta \gamma}=\frac{1}{2} \eta_{\beta \delta \epsilon \lambda} C_{\gamma \mu}{ }^{\epsilon \lambda} V^{\delta} V^{\mu},
$$

where the electric part has three non-zero components $E_{11}, E_{22}$ and $E_{12}$ while the magnetic part has $H_{13}$ and $H_{23}$. Thus we can write them in terms of unit spacelike vectors as

$$
\begin{aligned}
E_{\beta \gamma} & =\frac{1}{3}\left(\varepsilon_{I I}+2 \varepsilon_{I}\right)\left(K_{\beta} K_{\gamma}-\frac{1}{3} h_{\beta \gamma}\right)+\frac{1}{3}\left(\varepsilon_{I}+2 \varepsilon_{I I}\right)\left(L_{\beta} L_{\gamma}-\frac{1}{3} h_{\beta \gamma}\right) \\
& +\varepsilon_{K L}\left(K_{\beta} L_{\gamma}+K_{\gamma} L_{\beta}\right), \\
H_{\beta \gamma} & =H_{1}\left(S_{\beta} K_{\gamma}+S_{\gamma} K_{\beta}\right)+H_{2}\left(S_{\beta} L_{\gamma}+S_{\gamma} L_{\beta}\right) .
\end{aligned}
$$


The values of scalars $\varepsilon_{I}, \varepsilon_{I I}, \varepsilon_{K L}, H_{1}$ and $H_{2}$ in terms of metric functions are given in [23]. The elementary flatness condition states that a spacetime is locally isometric to Minkowski spacetime. The regularity condition necessary for the elementary flatness at the center $(r=0)$ implies that $\tilde{G}=0$ or it is a regular function of $r$ in the neighborhood of the center such that it approaches to zero as $r \approx 0$ [8, 30]. When $\tilde{G}=0$, Eq.(17) gives $\tilde{G}=0 \Leftrightarrow \Omega=0$ and in the neighborhood of center, we may write

$$
\tilde{G}=\sum_{m=3}^{\infty} \tilde{G}^{(m)}(\theta) r^{m}
$$

Substituting this value in Eq.(17), it follows that

$$
\Omega=\sum_{m=1}^{\infty} \Omega^{(m)}(t, \theta) r^{m}
$$

The condition of elementary flatness 31 implies that

$$
C \approx r \psi(t, \theta), \text { where } \psi(t, \theta) \approx B(t, 0, \theta) .
$$

Also, in the neighborhood of the center we can write

$$
B(t, r, \theta)=\sum_{m=0}^{\infty} B^{(m)}(t, \theta) r^{m}, \quad C(t, r, \theta)=\sum_{m=1}^{\infty} C^{(m)}(t, \theta) r^{m} .
$$

We discuss evolving axially symmetric dissipative dust fluid at the center and in its vicinity. In the first case, at the center we have $\Omega=0 \Leftrightarrow \tilde{G}=0$, i.e., the fluid is non-spinning. In the second case, we consider $\tilde{G}$ and $\Omega$ as regular functions of $r$, i.e., spinning fluid in the neighborhood of the center as defined in Eqs.(20) and (21).

Bel [33] introduced spatial tensors through orthogonal splitting of the Riemann tensor given as

$$
Y_{\beta \gamma}=R_{\beta \alpha \gamma \delta} V^{\alpha} V^{\delta}, \quad X_{\beta \gamma}=\frac{1}{2} \eta_{\beta \alpha}{ }^{\mu \nu} R_{\mu \nu \gamma \delta}^{*} V^{\alpha} V^{\delta}, \quad Z_{\beta \gamma}=\frac{1}{2} \epsilon_{\beta \mu \nu} R_{\delta \gamma}{ }^{\mu \nu} V^{\delta} .
$$

The scalars obtained from these tensors are called structure scalars which explain different physical processes during evolution of the system. we can define super energy and super-Poynting vector using the above spatial tensors as

$$
W=\frac{1}{2}\left[X^{\beta \gamma} X_{\beta \gamma}+Y^{\beta \gamma} Y_{\beta \gamma}\right]+Z^{\beta \gamma} Z_{\beta \gamma}
$$




$$
P_{\gamma}=\epsilon_{\gamma \mu \nu}\left(Y_{\delta \rho} Z^{\mu \rho}-X_{\delta \rho} Z^{\rho \mu}\right) g^{\nu \delta},
$$

where super energy having dimensions $L^{-4}$ can be interpreted as the energy per unit area and super-Poynting vector tells about the state of intrinsic gravitational radiation [34]. Sharif and Zunaira [23] studied structure scalars in the context of $f(R)$ gravity and evaluated super energy and super-Poynting vector for the model $R+\xi R^{2}$. We use this super-Poynting vector to confirm the presence of gravitational radiation in both cases. The super-Poynting vector in terms of spacelike vectors is given by

$$
P_{\mu}=P_{I} K_{\mu}+P_{I I} L_{\mu} .
$$

Here the scalars $P_{I}$ and $P_{I I}$ describe the flux of super energy which is a combination of gravitational radiation, heat dissipation and dark energy as

$$
\begin{aligned}
P_{I} & =2 H_{1} \varepsilon_{K L}+\frac{2 H_{2}}{3}\left(\varepsilon_{I}+2 \varepsilon_{I I}\right)+D_{1}, \\
P_{I I} & =2 H_{2} \varepsilon_{K L}-\frac{2 H_{1}}{3}\left(2 \varepsilon_{I}+\varepsilon_{I I}\right)+D_{2},
\end{aligned}
$$

where $D_{1}$ and $D_{2}$, given in Appendix $\mathbf{B}$, describe the effects of heat dissipation as well as $f(R)$ gravity. The scalars $H_{1}, H_{2}, \varepsilon_{I}, \varepsilon_{I I}$ and $\varepsilon_{K L}$, associated with magnetic and electric parts of the Weyl tensor are linked with gravitational radiation. If the values of these scalars obtained from evolution equations are non-zero then the above mentioned terms in the expression of super-Poyinting vector do not vanish indicating the existence of gravitational radiation.

For dissipative dust, the evolution equations for the expansion scalar, shear tensor and vorticity tensor obtained from the Ricci identities for $f(R)$ gravity are respectively, given by

$$
\begin{array}{r}
\Theta_{; \beta} V^{\beta}+\frac{1}{3} \Theta^{2}+2\left(\sigma^{2}-\Omega^{2}\right)+\frac{1}{F}\left[4 \pi \mu+F_{R} h^{\lambda \delta} \nabla_{\lambda} \nabla_{\delta} R\right]=0, \\
h_{\mu}^{\alpha} h_{\nu}^{\beta} \sigma_{\alpha \beta ; \gamma} V^{\gamma}+\sigma_{\mu}^{\alpha} \sigma_{\nu \alpha}+\frac{2}{3} \Theta \sigma_{\mu \nu}-\frac{1}{3}\left(2 \sigma^{2}+\Omega^{2}\right) h_{\mu \nu}+\omega_{\mu} \omega_{\nu} \\
+E_{\mu \nu}+\frac{1}{2 F} F_{R} \nabla_{\alpha} \nabla_{\beta} R\left(h_{\mu}^{\alpha} h_{\nu}^{\beta}-\frac{1}{3} h_{\mu \nu} h^{\alpha \beta}\right)=0, \\
h_{\mu}^{\alpha} h_{\nu}^{\beta} \Omega_{\alpha \beta ; \gamma} V^{\gamma}+\frac{2}{3} \Theta \Omega_{\mu \nu}-2 \sigma_{\alpha[\mu} \Omega_{\nu]}^{\alpha}=0 .
\end{array}
$$


The constraint equations are

$$
\begin{array}{r}
h_{\mu}^{\nu}\left(\frac{2}{3} \Theta_{; \nu}-h^{\delta \gamma} \sigma_{\nu \delta ; \gamma}\right)+\eta_{\mu}^{\nu \gamma \delta} V_{\delta} \omega_{\gamma ; \nu}-\frac{1}{F}\left[8 \pi q_{\mu}+F_{R} h_{\mu}^{\nu}\left(\nabla_{\nu} \nabla_{\gamma}\right) V^{\gamma}\right]=0 \\
h_{(\mu}^{\alpha} h_{\nu) \beta}\left(\sigma_{\alpha \delta}+\Omega_{\alpha \delta}\right)_{; \gamma} \eta^{\beta \kappa \gamma \delta} V_{\kappa}=H_{\mu \nu} .
\end{array}
$$

The conservation law gives

$$
\begin{aligned}
& h_{\mu}^{\nu} q_{\nu ; \alpha} V^{\alpha}+\left(\frac{4}{3} \Theta h_{\mu \nu}+\sigma_{\mu \nu}+\Omega_{\mu \nu}\right) q^{\nu}+\frac{1}{8 \pi}\left[(1-F) R_{\mu}^{\nu}\right. \\
& \left.+\nabla_{\nu} \nabla_{\mu} F+\delta_{\mu}^{\nu}\left(\frac{1}{2}(f-R)-\square F\right)\right]_{; \nu}=0 .
\end{aligned}
$$

The evolution and constraint equations for electric part of the Weyl tensor are given by

$$
\begin{aligned}
& h_{(\mu}^{\alpha} h_{\nu)}^{\beta} E_{\alpha \beta ; \delta} V^{\delta}+\Theta E_{\mu \nu}+h_{\mu \nu} E_{\alpha \beta} \sigma^{\alpha \beta}-3 E_{\alpha(\mu} \sigma_{\nu)}^{\alpha}+h_{(\mu}^{\alpha} \eta_{\nu)}^{\delta \gamma \kappa} V_{\delta} H_{\gamma \alpha ; \kappa} \\
- & E_{\delta(\mu} \Omega_{\nu)}^{\delta}=\frac{1}{F}\left\{-4 \pi \rho \sigma_{\mu \nu}+\frac{4 \pi}{3} q_{; \alpha}^{\alpha} h_{\mu \nu}-4 \pi h_{(\mu}^{\alpha} h_{\nu)}^{\beta} q_{\beta ; \alpha}\right\} \\
+ & \frac{F_{R}}{2 F} h_{(\mu}^{\alpha} h_{\nu)}^{\beta}\left\{\dot{R}\left(R_{\alpha \beta}-\frac{1}{3} R g_{\alpha \beta}\right)-\left(\nabla_{\alpha} R\right) R_{\beta \gamma} V^{\gamma}\right. \\
+ & \left.\nabla_{\alpha}\left(\nabla_{\gamma} \nabla_{\beta} R\right) V^{\gamma}-\left(\nabla_{\alpha} \nabla_{\beta} R\right) \cdot\right\} \\
& h_{\mu}^{\alpha} h^{\beta \nu} E_{\alpha \beta ; \nu}-\eta_{\mu}^{\delta \beta \kappa} V_{\delta} \sigma_{\beta}^{\gamma} H_{\kappa \gamma}+3 H_{\mu \nu} \omega^{\nu}=\frac{1}{F}\left\{\frac{8 \pi}{3} h_{\mu}^{\nu} \rho_{; \nu}-4 \pi\right. \\
\times \quad & \left.\left(\frac{2}{3} \Theta h_{\mu}^{\nu}-\sigma_{\mu}^{\nu}+3 \Omega_{\mu}^{\nu}\right) q_{\nu}\right\}+\frac{F_{R}}{2 F} h_{\mu}^{\alpha}\left\{\dot{R} R_{\alpha}^{\nu} V_{\nu}-\left(R_{\nu \gamma} V^{\nu} V^{\gamma}+\frac{1}{3} R\right)\right. \\
\times \quad & \left.R_{; \alpha}-(\square R)_{; \alpha}-\left(\nabla_{\alpha} \nabla_{\nu} R\right) \cdot V^{\nu}+h_{\gamma}^{\nu} \nabla_{\alpha}\left(\nabla_{\nu} \nabla^{\gamma} R\right)\right\}
\end{aligned}
$$

where $F=\frac{d f}{d R}$ and $F_{R}=\frac{d^{2} f}{d R^{2}}$. The value of Ricci scalar $R$, for the spacetime given in Eq.(31) is calculated as

$$
\begin{aligned}
R & =\frac{1}{r^{2} B^{2}+\tilde{G}^{2}}\left[\frac{2}{C}\left(C_{, \theta \theta}-r^{2} B^{2} \ddot{C}+2 \tilde{G} \dot{C}_{, \theta}-2 r^{2} B \dot{C} \dot{B}+r C^{\prime}\right)-\frac{2}{B^{2} C}\right. \\
& \times\left[C^{\prime \prime}\left(r^{2} B^{2}+\tilde{G}^{2}\right)-\tilde{G} \tilde{G}^{\prime} C^{\prime}-\tilde{G} C \tilde{G}^{\prime \prime}\right]+\frac{2}{B}\left(2 \tilde{G} \dot{B}_{, \theta}+r^{2} B^{\prime \prime}+B_{, \theta \theta}\right) \\
& \left.-\frac{2 \tilde{G}^{2} B^{\prime} C^{\prime}}{B^{3} C}\right]-\frac{1}{2 B^{3} C\left(r^{2} B^{2}+\tilde{G}^{2}\right)^{2}}\left[4 \tilde{G} C \tilde{G}^{\prime} B^{\prime}\left(3 r^{2} B^{2}+\tilde{G}^{2}\right)+8 r^{2} B^{4} \dot{C} \dot{B}\right.
\end{aligned}
$$




$$
\begin{array}{ll}
\times & \left(r^{2} B^{2}+2 \tilde{G}^{2}\right)+\left(r^{2} B^{2}+\tilde{G}^{2}\right)\left(4 B^{3} r^{2} C \dot{B}^{2}-4 B^{2} C C^{\prime} B^{\prime}\right)+4 B^{3} C r^{2} \\
\times & \left(r^{2} B^{\prime 2}+B_{, \theta}^{2}\right)+4 \tilde{G}_{, \theta}\left(B^{3} \tilde{G} C_{, \theta}-B^{5} r^{2} \dot{C}-B^{4} r^{2} C \dot{B}+B^{2} C \tilde{G} B_{, \theta}\right) \\
+ & \left.4 B_{, \theta} B^{2} \tilde{G}\left(2 C r^{2} B \dot{B}-\tilde{G} C_{, \theta}-\tilde{G}^{2} \dot{C}\right)+8 \tilde{G} C r B^{3} G^{\prime}-4 B^{3} C \tilde{G}^{2}\right] .
\end{array}
$$

From Eqs.(20) and (23), the behavior of $R$ near the center is given by

$$
R(t, r, \theta)=\sum_{m=-2}^{\infty} R^{(m)}(t, \theta) r^{m} .
$$

It gives $R \rightarrow \infty$ as $r \rightarrow 0$. This implies that there is a singularity at $r=0$ or at the center of considered configuration (which makes the curvature scalar infinite) [9]. In this paper, we use the $f(R)$ model given as

$$
f(R)=\lambda R+\xi R^{2},
$$

where $\lambda$ and $\xi$ are positive real numbers. In the limit $\lambda \rightarrow 1$ and $\xi \rightarrow 0$, this reduces to GR. Any model in which $f(R) \propto R^{2}$ can explain the inflationary scenario but cannot tell about the recent cosmic expansion. However, the inclusion of linear term in $R$ causes the inflation to end when $R^{2}$ term is smaller than the linear term (this occurs by a reheating stage in which gravitational particles are produced due to oscillations in $R$ ) [35]. Consequently, it can lead to expansion of the universe. This model being quadratic can also explain dark matter and dark energy [36]. This reduces to Starobinsky inflationary model when $\lambda \rightarrow 1$, which estimates nearly flat spectrum of gravitational waves and is also consistent with the temperature anisotropies measured by CMBR [35].

\section{$3 \quad$ Non-Spinning Dissipative Dust}

In this section, we deal with the non-spinning case. Using the condition $\tilde{G}=0 \Leftrightarrow \Omega=0$, Eqs.(12) and (14) reduce to the following form

$$
\Theta=2 \frac{\dot{B}}{B}+\frac{\dot{C}}{C}, \quad \sigma_{I}=\sigma_{I I}=\tilde{\sigma}=\frac{\dot{B}}{B}+\frac{\dot{C}}{C} .
$$

The general evolution equations obtained through Ricci and Bianchi identities are given in Appendix A while the contracted equations are in Appendix B. The equations $(\underline{\mathrm{B} 5})-(\underline{\mathrm{B} 8})$ along with $\Omega=0=\tilde{G}$ give

$$
\frac{1}{3 B}\left\{2 \Theta^{\prime}-\tilde{\sigma}^{\prime}-\tilde{\sigma} \frac{3 C^{\prime}}{C}\right\}=\frac{1}{(\lambda+2 \xi R)}\left\{8 \pi q_{I}+2 \xi K^{\nu}\left(\nabla_{\nu} \nabla_{\lambda} R\right) V^{\lambda}\right\},
$$




$$
\begin{array}{r}
\frac{1}{3 r B}\left\{2 \Theta_{, \theta}-\tilde{\sigma}_{, \theta}-\tilde{\sigma} \frac{3 C_{, \theta}}{C}\right\}=\frac{1}{(\lambda+2 \xi R)}\left\{8 \pi q_{I I}+2 \xi L^{\nu}\left(\nabla_{\nu} \nabla_{\lambda} R\right) V^{\lambda}\right\}, \\
H_{1}=-\frac{\tilde{\sigma}}{2 r B}\left(\frac{\tilde{\sigma}, \theta}{\tilde{\sigma}}+\frac{C_{, \theta}}{C}\right)=-\frac{(\tilde{\sigma} C)_{, \theta}}{2 r B C} \\
H_{2}=\frac{\tilde{\sigma}}{2 B}\left(\frac{\tilde{\sigma}^{\prime}}{\tilde{\sigma}}+\frac{C^{\prime}}{C}\right)=\frac{(\tilde{\sigma} C)^{\prime}}{2 B C} .
\end{array}
$$

Using Eq.(37) in Eqs.(38) and (39), we obtain

$$
\begin{gathered}
q_{I}=-\frac{1}{8 \pi}\left\{2 \xi K^{\nu}\left(\nabla_{\nu} \nabla_{\kappa}\right) V^{\kappa}\right\}+\frac{(\lambda+2 \xi R)}{8 \pi B}\left\{\left(\frac{\dot{B}}{B}\right)^{\prime}-\frac{\dot{B}}{B} \frac{C^{\prime}}{C}+\frac{\dot{C}^{\prime}}{C}\right\}, \\
q_{I I}=-\frac{1}{8 \pi}\left\{2 \xi L^{\nu}\left(\nabla_{\nu} \nabla_{\kappa}\right) V^{\kappa}\right\}+\frac{(\lambda+2 \xi R)}{8 \pi r B}\left\{\left(\frac{\dot{B}}{B}\right)_{, \theta}-\frac{\dot{B}}{B} \frac{C_{, \theta}}{C}+\frac{\dot{C}_{, \theta}}{C}\right\} .
\end{gathered}
$$

These show the presence of heat flux as well as the effect of $f(R)$ curvature terms on dissipation. Using Eqs.(42) and (43) in (40) and (41), we obtain

$$
\begin{aligned}
& H_{1}=-\frac{1}{r B}\left(\frac{\dot{B}}{B}\right)_{, \theta}+\frac{1}{\lambda+2 \xi R}\left\{4 \pi q_{I I}+\xi L^{\nu}\left(\nabla_{\nu} \nabla_{\kappa}\right) V^{\kappa}\right\}, \\
& H_{2}=-\frac{1}{B}\left(\frac{\dot{B}}{B}\right)^{\prime}-\frac{1}{\lambda+2 \xi R}\left\{4 \pi q_{I}+\xi K^{\nu}\left(\nabla_{\nu} \nabla_{\kappa}\right) V^{\kappa}\right\} .
\end{aligned}
$$

These equations indicate that $H_{1}$ and $H_{2}$ have the contribution of heat flux and $f(R)$ gravity. If we assume $B$ and $C$ explicit functions of $t$ and $(r, \theta)$ as $B(t, r, \theta)=T(t) \tilde{B}(r, \theta)$ and $C(t, r, \theta)=T(t) \tilde{C}(r, \theta)$ then Eqs.(42)-(45) become

$$
\begin{aligned}
q_{I} & =-\frac{1}{8 \pi}\left\{2 \xi K^{\nu}\left(\nabla_{\nu} \nabla_{\kappa}\right) V^{\kappa}\right\}, \\
q_{I I} & =-\frac{1}{8 \pi}\left\{2 \xi L^{\nu}\left(\nabla_{\nu} \nabla_{\kappa}\right) V^{\kappa}\right\}, \\
H_{1} & =\frac{1}{\lambda+2 \xi R}\left\{4 \pi q_{I I}+\xi L^{\nu}\left(\nabla_{\nu} \nabla_{\kappa}\right) V^{\kappa}\right\}, \\
H_{2} & =-\frac{1}{\lambda+2 \xi R}\left\{4 \pi q_{I}+\xi K^{\nu}\left(\nabla_{\nu} \nabla_{\kappa}\right) V^{\kappa}\right\} .
\end{aligned}
$$


In the limit $\xi \rightarrow 0$, we obtain the results of GR. From Eq.(36) we see that when $r \rightarrow 0, R \rightarrow \infty$ and Eq.(48) and (49) give

$$
H_{1}=0, H_{2}=0 \text {. }
$$

Thus Eqs.(24)-(26) indicate that the scalars of super-Poynting vector and hence the super-Poynting vector contains no contribution due to gravitational radiation. It is mentioned here that the non-spinning dissipative dust is also not gravitationally radiating in GR. To find the values of $\varepsilon_{I}, \varepsilon_{I I}$ and $\varepsilon_{K L}$, we consider Eqs.(Bי) and (B10) given by

$$
\begin{aligned}
& \frac{1}{3} \dot{\varepsilon}_{I}+\frac{1}{3} \varepsilon_{I} \Theta+\frac{1}{3} \varepsilon_{I I} \tilde{\sigma}=0, \\
& \frac{1}{3} \dot{\varepsilon}_{I I}+\frac{1}{3} \varepsilon_{I I} \Theta+\frac{1}{3} \varepsilon_{I} \tilde{\sigma}=0,
\end{aligned}
$$

where we have used the values of $H_{1}, H_{2}$ and $R$ at $r \rightarrow 0$. Solving the above two equations simultaneously, we obtain

$$
\begin{aligned}
\varepsilon_{I} & =\frac{1}{2 B}\left\{\frac{1}{B^{2} C^{2}}+1\right\} \\
\varepsilon_{I I} & =\frac{1}{2 B}\left\{\frac{1}{B^{2} C^{2}}-1\right\} .
\end{aligned}
$$

As $C \rightarrow 0$ when $r \rightarrow 0$ implying that $\varepsilon_{I}$ and $\varepsilon_{I I}$ approaches to infinity. Similarly, the value of $\varepsilon_{K L}$ obtained from Eq.(B12) is given by

$$
\varepsilon_{K L}=\frac{1}{B}
$$

\section{Spinning Dissipative Dust}

In this case, we take non-zero vorticity scalar (spinning fluid), which is a regular function of $r$ such that it vanishes at the center. Moreover, all geometrical and physical variables are regular at $r \approx 0$. Using Eqs.(20) and (23) in Eq.(15), it follows that

$$
\sigma_{I}-\sigma_{I I}=\sum_{m=4}^{\infty}\left[\sigma_{I}^{(m)}(t, \theta)-\sigma_{I I}^{(m)}(t, \theta)\right] r^{m} .
$$


Similarly, from Eq.(12) we can write

$$
\Theta=\sum_{m=0}^{\infty} \Theta^{(m)}(t, \theta) r^{m}
$$

Contraction of Eq.(B14) with $K^{\mu}$ and $L^{\mu}$ give the following equations

$$
\begin{array}{r}
\Omega q_{I I}+\frac{\kappa T^{\prime}}{\tau B}+\left\{\frac{1}{\tau}+\frac{1}{2} D_{t}\left[\ln \left(\frac{\tau}{\kappa T^{2}}\right)\right]-\frac{5}{6} \Theta\right\} q_{I}-\frac{q_{I} \sigma_{I}}{3}+D_{3}=0, \\
-\Omega q_{I}+\frac{\kappa L^{\mu} T_{, \mu}}{\tau}+\left\{\frac{1}{\tau}+\frac{1}{2} D_{t}\left[\ln \left(\frac{\tau}{\kappa T^{2}}\right)\right]-\frac{5}{6} \Theta\right\} q_{I I}-\frac{q_{I I} \sigma_{I I}}{3}+D_{4}=0,(58
\end{array}
$$

where $D_{t}$ represents covariant derivative with respect to time and $\tau$ is the relaxation time. Here $D_{3}$ and $D_{4}$ indicate the terms due to $f(R)$ extra degrees of freedom given in (B18) and (B19), respectively. From Eqs.(B18) and (B19), the behavior of $D_{3}$ and $D_{4}$ is given by

$$
D_{3}=\sum_{m=-5}^{\infty} D_{3}^{(m)}(t, \theta) r^{m}, D_{4}=\sum_{m=-5}^{\infty} D_{4}^{(m)}(t, \theta) r^{m}
$$

Since we have assumed that all the variables are regular, so is the dissipation scalars $q_{I}$ and $q_{I I}$. Let us take

$$
q_{I}=\sum_{m=0}^{\infty} q_{I}^{(m)}(t, \theta) r^{m}, \quad q_{I I}=\sum_{m=0}^{\infty} q_{I I}^{(m)}(t, \theta) r^{m}
$$

near the center. Considering the terms of $r^{(0)}$ in Eq.(57), we have

$$
\begin{array}{r}
\left\{\frac{1}{\tau}+\frac{1}{2} D_{t}\left[\ln \left(\frac{\tau}{\kappa\left(T^{(0)}\right)^{2}}\right)\right]-\frac{5}{6} \Theta^{(0)}\right\} \\
q_{I}^{(0)}(t, \theta)+D_{3}^{(0)}(t, \theta) \\
+\frac{\kappa}{\tau B^{(0)}} T^{(1)}(t, \theta)=0
\end{array}
$$

where it is assumed that in the neighborhood of center, $T$ (temperature) has the same behavior as $q_{I}$ and $q_{I I}$, i.e.,

$$
T=\sum_{m=0}^{\infty} T^{(m)}(t, \theta) r^{m}
$$


Similarly, the coefficients of $r^{(0)}$ in Eq.(58) give

$$
\begin{array}{r}
\left\{\frac{1}{\tau}+\frac{1}{2} D_{t}\left[\ln \left(\frac{\tau}{\kappa\left(T^{(0)}\right)^{2}}\right)\right]-\frac{5}{6} \Theta^{(0)}\right\} \\
q_{I I}^{(0)}(t, \theta)+D_{4}^{(0)}(t, \theta) \\
+\frac{\kappa}{\tau B^{(0)}} T_{, \theta}^{(0)}(t, \theta)=0 .
\end{array}
$$

From the above equations, it is clear that $q_{I}^{(0)}(t, \theta)$ and $q_{I I}^{(0)}(t, \theta)$ are nonzero and contain the effects of $f(R)$ terms. Consequently, when $r \approx 0$, the dissipation scalars are non-zero showing that there is heat dissipation at the center.

Next excluding the singularities of the scalars $\varepsilon_{I}, \varepsilon_{I I}, \varepsilon_{K L}, H_{1}$ and $H_{2}$ at the center, we may consider

$$
\begin{array}{r}
\varepsilon_{I}=\sum_{m=0}^{\infty} \varepsilon_{I}^{(m)}(t, \theta) r^{m}, \quad \varepsilon_{I I}=\sum_{m=0}^{\infty} \varepsilon_{I I}^{(m)}(t, \theta) r^{m}, \quad \varepsilon_{K L}=\sum_{m=0}^{\infty} \varepsilon_{K L}^{(m)}(t, \theta) r^{m},( \\
H_{1}=\sum_{m=0}^{\infty} H_{1}^{(m)}(t, \theta) r^{m}, \quad H_{2}=\sum_{m=0}^{\infty} H_{2}^{(m)}(t, \theta) r^{m} .
\end{array}
$$

Using these expressions in Eqs.(B1)-(B3) and comparison of the coefficients of $r^{(0)}$ on both sides respectively, give

$$
\begin{aligned}
\varepsilon_{I}^{(0)}(t, \theta) & =-D_{5}^{(0)}(t, \theta), \\
\varepsilon_{K L}^{(0)}(t, \theta) & =-D_{6}^{(0)}(t, \theta), \\
\varepsilon_{I I}^{(0)}(t, \theta) & =-D_{7}^{(0)}(t, \theta),
\end{aligned}
$$

where $D_{5}, D_{6}$ and $D_{7}$ describe the effects of $f(R)$ curvature terms given in Appendix B. The behavior of these terms near the center is given by

$D_{5}=\sum_{m=-2}^{\infty} D_{5}^{(m)}(t, \theta) r^{m}, \quad D_{6}=\sum_{m=-2}^{\infty} D_{6}^{(m)}(t, \theta) r^{m}, \quad D_{7}=\sum_{m=-2}^{\infty} D_{7}^{(m)}(t, \theta) r^{m}$.

From Eqs. (66) - (68) , it is obvious that scalars of the electric part of the Weyl tensor do not vanish at the center because their zeroth order coefficients are non-zero. Also, $D_{5}, D_{6}$ and $D_{7}$ approach to zero as $\xi \rightarrow 0$, implying that $D_{5}^{(0)}, D_{6}^{(0)}$ and $D_{7}^{(0)}$ become zero which in turn give $\varepsilon_{I}^{(0)}, \varepsilon_{K L}^{(0)}$ and $\varepsilon_{I I}^{(0)}$ zero. Further assuming that these scalars are of class $C^{\omega}$, we have zero value of $\varepsilon_{I}, \varepsilon_{K L}$ and $\varepsilon_{I I}$ for the whole configuration. Thus Eqs.(24) and (25) show 
that the matter is not gravitationally radiating, which is the case of GR. Comparing the coefficients of $r^{(0)}$, Eqs. (B9) and (B10) imply that

$$
\begin{array}{r}
-H_{1, \theta}^{(0)}-H_{1}^{(0)} \frac{C_{, \theta}^{(1)}}{C^{(1)}}+\frac{H_{2}^{(0)}}{B^{(0)}}-H_{2}^{(0)}=D_{8}^{(0)}, \\
H_{2}^{(0)}+H_{1}^{(0)}\left[\frac{C_{, \theta}^{(1)}}{C^{(1)}}-\frac{B_{, \theta}^{(0)}}{B^{(0)}}\right]=D_{9}^{(0)} .
\end{array}
$$

The terms $D_{8}$ and $D_{9}$ are given in Appendix $\mathbf{B}$ and their behavior in the neighborhood of the center can be written as

$$
D_{8}=\sum_{m=-2}^{\infty} D_{8}^{(m)}(t, \theta) r^{m}, \quad D_{9}=\sum_{m=-2}^{\infty} D_{9}^{(m)}(t, \theta) r^{m} .
$$

Using the value of $H_{2}^{(0)}$ from Eq.(70) into (69), we obtain

$$
H_{1, \theta}^{(0)}+H_{1}^{(0)}\left[\frac{C_{, \theta}^{(1)}}{C^{(1)}}+\left(1-\frac{1}{B^{(0)}}\right)\left\{\frac{C_{, \theta}^{(1)}}{C^{(1)}}-\frac{B_{, \theta}^{(0)}}{B^{(0)}}\right\}\right]=-D_{8}^{(0)}-D_{9}^{(0)}
$$

which is a first order linear differential equation and its solution is given by

$$
H_{1}^{(0)}=-C^{(1)}\left(\frac{C^{(1)}}{B^{(0)}}\right)^{\left(1-\frac{1}{B^{(0)}}\right)} \int C^{(1)}\left(\frac{C^{(1)}}{B^{(0)}}\right)^{\left(1-\frac{1}{B^{(0)}}\right)}\left(D_{8}^{(0)}+D_{9}^{(0)}\right) d \theta \text {. }
$$

Substituting $H_{1}^{(0)}$ in Eq.(70), we obtain the value of $H_{2}^{(0)}$. Thus $H_{1}^{(0)}, H_{2}^{(0)}$ $\varepsilon_{I}^{(0)}, \varepsilon_{I I}^{(0)}$ and $\varepsilon_{K L}^{(0)}$ are non-zero implying that the scalars $H_{1}, H_{2}, \varepsilon_{I}, \varepsilon_{I I}$ and $\varepsilon_{K L}$ do not vanish when $r \approx 0$. Assuming that the scalars defined in Eqs. (60), (64) and (65) are of class $C^{\omega}$, thus we have non-vanishing values of these scalars for the whole configuration. Hence the super-Poynting vector shows that the spinning dust is producing gravitational radiation in contrast to GR where the fluid is not gravitationally radiating for this case.

\section{Final Remarks}

The process of gravitational radiation produced by self-gravitating objects has attracted many researchers due to their fascinating features. If we could observe these waves it may open new horizons to solve different cosmic issues. 
This paper is devoted to study the gravitational radiation produced by dissipative dust cloud in the background of $f(R)$ gravity. It is assumed that the matter configuration is axially symmetric and geodesic. We have discussed the values of scalars in super-Poynting vector to assure the presence of gravitational radiation. For this purpose, we have used evolution equations of non-spinning and spinning fluids. In the non-spinning case, we have found that the scalars $q_{I}$ and $q_{I I}$ are non-zero at $r=0$ leading to non-zero heat dissipation. This implies that there is heat flux at the center in the form of radiation during the evolution process. Equations (48) and (49) indicate that the scalars associated with magnetic part of the Weyl tensor are zero. It turns out that geodesic dust with dissipation do not produce gravitational radiation at the center.

In the second case (spinning fluid), a combination of conservation and transport equations imply that the values of dissipation scalars are nonzero when $r \approx 0$. Similarly, near the axis of symmetry, we have discussed the coefficients of $r^{m}$ in Eqs.(64) and (65) and found that the magnetic as well as electric parts of the Weyl tensor are non-zero. In this case, all scalars $q_{I}, q_{I I}, \varepsilon_{I}, \varepsilon_{I I}, \varepsilon_{K L}, H_{1}$ and $H_{2}$ depend upon $f(R)$ model. Thus we conclude that the matter under consideration is gravitationally radiating for non-vanishing vorticity in the framework of $f(R)$ gravity. It is worthwhile to mention here that our results reduce to GR in the limit $\xi \rightarrow 0[8$. For nonspinning case, Eqs.(46)-(49) give zero values of $H_{1}$ and $H_{2}$ in the limit $\xi \rightarrow 0$ while for spinning case, Eqs.(66)-(68) yield zero value of $\varepsilon_{I}, \varepsilon_{K L}$ and $\varepsilon_{I I}$ implying that the gravitational part of super-Poynting vector is equal to zero in both cases. In GR, both cases imply that the matter under consideration could not act as a source of gravitational radiation.

Thus for a spinning celestial object having axially symmetry there are no gravitational waves at the center (because the center is on spinning axis). Also, there is a singularity at the center. The gravitational radiation is produced in the spacetime fabric due to the disturbance followed by the spinning of celestial object. Finally, we can conclude that an astrophysical object having axially symmetry consisting of dissipative dust can emit gravitational radiation in the framework of $f(R)$ gravity. This is significant as there is no gravitational radiation for axially symmetric spinning celestial object in GR. 


\section{Appendix A}

The time propagation equations for the expansion scalar, shear tensor and vorticity tensor obtained from the Ricci identities in $f(R)$ gravity model $f(R)=\lambda R+\xi R^{2}$ are respectively, given by

$$
\begin{array}{r}
\Theta_{; \beta} V^{\beta}+\frac{1}{3} \Theta^{2}+2\left(\sigma^{2}-\Omega^{2}\right)+\frac{1}{(\lambda+2 \xi R)}\left[4 \pi \mu+2 \xi h^{\lambda \delta} \nabla_{\lambda} \nabla_{\delta} R\right]=0 \\
h_{\mu}^{\alpha} h_{\nu}^{\beta} \sigma_{\alpha \beta ; \gamma} V^{\gamma}+\sigma_{\mu}^{\alpha} \sigma_{\nu \alpha}+\frac{2}{3} \Theta \sigma_{\mu \nu}-\frac{1}{3}\left(2 \sigma^{2}+\Omega^{2}\right) h_{\mu \nu}+\omega_{\mu} \omega_{\nu} \\
+E_{\mu \nu}+\frac{1}{(\lambda+2 \xi R)} \xi \nabla_{\alpha} \nabla_{\beta} R\left(h_{\mu}^{\alpha} h_{\nu}^{\beta}-\frac{1}{3} h_{\mu \nu} h^{\alpha \beta}\right)=0 \\
h_{\mu}^{\alpha} h_{\nu}^{\beta} \Omega_{\alpha \beta ; \gamma} V^{\gamma}+\frac{2}{3} \Theta \Omega_{\mu \nu}-2 \sigma_{\alpha[\mu} \Omega_{\nu]}^{\alpha}=0 .
\end{array}
$$

The constraint equations are

$$
\begin{array}{r}
h_{\mu}^{\nu}\left(\frac{2}{3} \Theta_{; \nu}-h^{\delta \gamma} \sigma_{\nu \delta ; \gamma}\right)+\eta_{\mu}^{\nu \gamma \delta} V_{\delta} \omega_{\gamma ; \nu}-\frac{1}{(\lambda+2 \xi R)}\left[8 \pi q_{\mu}+2 \xi h_{\mu}^{\nu}\left(\nabla_{\nu} \nabla_{\gamma}\right) V^{\gamma}\right]=0(\mathrm{~A}) \\
h_{(\mu}^{\alpha} h_{\nu) \beta}\left(\sigma_{\alpha \delta}+\Omega_{\alpha \delta}\right)_{; \gamma} \eta^{\beta \kappa \gamma \delta} V_{\kappa}=H_{\mu \nu}(\mathrm{A} 5)
\end{array}
$$

The conservation law gives

$$
\begin{aligned}
& h_{\mu}^{\nu} q_{\nu ; \alpha} V^{\alpha}+\left(\frac{4}{3} \Theta h_{\mu \nu}+\sigma_{\mu \nu}+\Omega_{\mu \nu}\right) q^{\nu}+\frac{1}{8 \pi}\left[\left\{\nabla^{\nu} \nabla_{\mu}(\lambda+2 \xi R)-2 \xi R R_{\mu}^{\nu}\right\}_{; \nu}\right. \\
& \left.-\delta_{\mu}^{\nu}\left\{2 \xi(\square R)_{; \nu}+\frac{1}{2}\left(\xi R^{2}\right)_{; \nu}\right\}\right]=0 .
\end{aligned}
$$

The evolution and constraint equations for electric part of the Weyl tensor are given by

$$
\begin{aligned}
& h_{(\mu}^{\alpha} h_{\nu)}^{\beta} E_{\alpha \beta ; \delta} V^{\delta}+\Theta E_{\mu \nu}+h_{\mu \nu} E_{\alpha \beta} \sigma^{\alpha \beta}-3 E_{\alpha(\mu} \sigma_{\nu)}^{\alpha}+h_{(\mu}^{\alpha} \eta_{\nu)}^{\delta \gamma \kappa} V_{\delta} H_{\gamma \alpha ; \kappa} \\
- & E_{\delta(\mu} \Omega_{\nu)}^{\delta}=\frac{1}{(\lambda+2 \xi R)}\left\{-4 \pi \rho \sigma_{\mu \nu}+\frac{4 \pi}{3} q_{; \alpha}^{\alpha} h_{\mu \nu}-4 \pi h_{(\mu}^{\alpha} h_{\nu)}^{\beta} q_{\beta ; \alpha}\right\} \\
+ & \frac{\xi}{(\lambda+2 \xi R)} h_{(\mu}^{\alpha} h_{\nu)}^{\beta}\left\{\dot{R}\left(R_{\alpha \beta}-\frac{1}{3} R g_{\alpha \beta}\right)-\left(\nabla_{\alpha} R\right) R_{\beta \gamma} V^{\gamma}\right. \\
+\quad & \left.\nabla_{\alpha}\left(\nabla_{\gamma} \nabla_{\beta} R\right) V^{\gamma}-\left(\nabla_{\alpha} \nabla_{\beta} R\right)^{\cdot}\right\}, \\
& h_{\mu}^{\alpha} h^{\beta \nu} E_{\alpha \beta ; \nu}-\eta_{\mu}^{\delta \beta \kappa} V_{\delta} \sigma_{\beta}^{\gamma} H_{\kappa \gamma}+3 H_{\mu \nu} \omega^{\nu}=\frac{1}{(\lambda+2 \xi R)}\left\{\frac{8 \pi}{3} h_{\mu}^{\nu} \rho_{; \nu}-4 \pi\right.
\end{aligned}
$$




$$
\begin{array}{ll}
\times & \left.\left(\frac{2}{3} \Theta h_{\mu}^{\nu}-\sigma_{\mu}^{\nu}+3 \Omega_{\mu}^{\nu}\right) q_{\nu}\right\}+\frac{\xi}{(\lambda+2 \xi R)} h_{\mu}^{\alpha}\left\{\dot{R} R_{\alpha}^{\nu} V_{\nu}-\left(R_{\nu \gamma} V^{\nu} V^{\gamma}+\frac{1}{3} R\right)\right. \\
\left.\times \quad R_{; \alpha}-(\square R)_{; \alpha}-\left(\nabla_{\alpha} \nabla_{\nu} R\right) \cdot V^{\nu}+h_{\gamma}^{\nu} \nabla_{\alpha}\left(\nabla_{\nu} \nabla^{\gamma} R\right)\right\} .
\end{array}
$$

\section{Appendix B}

Here we contract the equations in appendix A with spacelike vectors to get scalar equations. Equation (A1) remains the same. Contraction of Eq.(A2) with $K^{\mu} K^{\nu}, K^{\mu} L^{\nu}$ and with $L^{\mu} L^{\nu}$ gives

$$
\begin{aligned}
& \sigma_{I, \lambda} V^{\lambda}+\frac{1}{9} \sigma_{I}^{2}+\frac{2}{3} \Theta \sigma_{I}-\frac{2}{9} \sigma_{I I}\left(\sigma_{I}+\sigma_{I I}\right)+\varepsilon_{I}+\frac{3 \xi}{(\lambda+2 \xi R)} \\
\times & \left(K^{\alpha} K^{\beta}-\frac{1}{3} h_{\mu \nu} K^{\mu} K^{\nu} h^{\alpha \beta}\right) \nabla_{\alpha} \nabla_{\beta} R=\Omega^{2}, \\
& \frac{1}{3}\left(\sigma_{I}-\sigma_{I I}\right) \Omega+\varepsilon_{K L}+\frac{\xi}{(\lambda+2 \xi R)}\left(K^{\alpha} L^{\beta}\right. \\
- & \left.\frac{1}{3} h_{\mu \nu} K^{\mu} L^{\nu} h^{\alpha \beta}\right) \nabla_{\alpha} \nabla_{\beta} R=0, \\
& \sigma_{I I, \lambda} V^{\lambda}+\frac{1}{9} \sigma_{I I}^{2}+\frac{2}{3} \Theta \sigma_{I I}-\frac{2}{9} \sigma_{I}\left(\sigma_{I}+\sigma_{I I}\right)+\varepsilon_{I I}+\frac{3 \xi}{(\lambda+2 \xi R)} \\
\times & \left(L^{\alpha} L^{\beta}-\frac{1}{3} h_{\mu \nu} L^{\mu} L^{\nu} h^{\alpha \beta}\right) \nabla_{\alpha} \nabla_{\beta} R=\Omega^{2} .
\end{aligned}
$$

Contracting Eq. (A3) with $K^{\mu} L^{\nu}$ yields

$$
\Omega_{, \lambda} V^{\lambda}+\frac{1}{3}\left(2 \Theta+\sigma_{I}+\sigma_{I I}\right) \Omega=0 .
$$

Contraction of Eq.(A4) with $K^{\mu}$ and $L^{\mu}$ gives

$$
\begin{array}{r}
\frac{-1}{\sqrt{r^{2} B^{2}+\tilde{G}^{2}}}\left\{\Omega_{, \theta}+\tilde{G} \dot{\Omega}+\Omega\left(\frac{\tilde{G} \dot{C}}{C}+\frac{C_{, \theta}}{C}\right)\right\}+\frac{1}{3 B}\left[2 \Theta^{\prime}-\sigma_{I}^{\prime}\right. \\
\left.-\sigma_{I}\left\{\frac{2 C^{\prime}}{C}+\frac{\left(r^{2} B^{2}+\tilde{G}^{2}\right)^{\prime}}{2\left(r^{2} B^{2}+\tilde{G}^{2}\right)}\right\}-\sigma_{I I}\left\{\frac{2 C^{\prime}}{C}-\frac{\left(r^{2} B^{2}+\tilde{G}^{2}\right)^{\prime}}{2\left(r^{2} B^{2}+\tilde{G}^{2}\right)}\right\}\right]
\end{array}
$$




$$
\begin{aligned}
= & \frac{1}{\lambda+2 \xi R}\left\{8 \pi q_{I}+2 \xi K^{\nu} \nabla_{\nu} \nabla_{\lambda} V^{\lambda}\right\}, \\
& \frac{1}{B}\left(\Omega^{\prime}+\Omega \frac{C^{\prime}}{C}\right)+\frac{1}{3\left(\sqrt{r^{2} B^{2}+\tilde{G}^{2}}\right)}\left[\left(2 \Theta-\sigma_{I I}\right)_{, \theta}+\tilde{G}\left(2 \Theta-\sigma_{I I}\right)\right. \\
+ & \left.\sigma_{I}\left\{\frac{B_{, \theta}}{B}-\frac{C_{, \theta}}{C}+\tilde{G}\left(\frac{\dot{B}}{B}-\frac{\dot{C}}{C}\right)\right\}-\sigma_{I I}\left\{\frac{B_{, \theta}}{B}+\frac{2 C_{, \theta}}{C}+\tilde{G}\left(\frac{\dot{B}}{B}+\frac{2 \dot{C}}{C}\right)\right\}\right] \\
= & \frac{1}{\lambda+2 \xi R}\left\{8 \pi q_{I I}+\xi L^{\nu}\left(\nabla_{\nu} \nabla_{\lambda} R\right) V^{\lambda}\right\} .
\end{aligned}
$$

Contraction of Eq. (A5) with $K^{\mu} S^{\nu}$ and $L^{\mu} S^{\nu}$ leads to

$$
\begin{aligned}
H_{1} & =\frac{-1}{2 B}\left[\Omega^{\prime}-\Omega\left\{\frac{C^{\prime}}{C}-\frac{\tilde{G} \tilde{G}^{\prime}}{2\left(\sqrt{r^{2} B^{2}+\tilde{G}^{2}}\right)}\right\}\right] \frac{1}{6\left(\sqrt{r^{2} B^{2}+\tilde{G}^{2}}\right)} \\
& \times\left[\left(2 \sigma_{I}+\sigma_{I I}\right)_{, \theta}+\sigma_{I}\left\{\frac{B_{, \theta}}{B}+\frac{C_{, \theta}}{C}-\tilde{G}\left(\frac{\dot{B}}{B}-\frac{\dot{C}}{C}\right)\right\}\right. \\
& \left.-\sigma_{I I}\left\{\frac{B_{, \theta}}{B}-\frac{2 C_{, \theta}}{C}+\tilde{G}\left(\frac{2 \dot{B}}{B}-\frac{2 \dot{C}}{C}\right)\right\}\right], \\
H_{2} & =\frac{1}{6 B}\left[\left(\sigma_{I}+2 \sigma_{I I}\right)^{\prime}+\sigma_{I}\left\{\frac{2 C^{\prime}}{C}-\frac{(r B)(r B)^{\prime}}{r^{2} B^{2}+\tilde{G}^{2}}\right\}\right. \\
& \left.+\sigma_{I I}\left\{\frac{C^{\prime}}{C}+\frac{2(r B)(r B)^{\prime}+3 \tilde{G} \tilde{G}^{\prime}}{2\left(r^{2} B^{2}+\tilde{G}^{2}\right)}\right\}\right]-\frac{1}{2 \sqrt{r^{2} B^{2}+\tilde{G}^{2}}} \\
& \times\left[\Omega_{, \theta}-\Omega\left\{\frac{C_{, \theta}}{C}+\tilde{G}\left(\frac{\dot{B}}{B}+\frac{\dot{C}}{C}\right)\right\}\right] .
\end{aligned}
$$

Contraction of Eq.(A7) with $K^{\mu} K^{\nu}, L^{\mu} L^{\nu}, S^{\mu} S^{\nu}$ and $K^{\mu} L^{\nu}$ yield

$$
\begin{aligned}
& \frac{1}{3} \dot{\varepsilon}_{I}+\frac{1}{9} \varepsilon_{I}\left(3 \Theta+\sigma_{I I}-\sigma_{I}\right)+\frac{1}{9} \varepsilon_{I I}\left(2 \sigma_{I I}+\sigma_{I}\right)-\Omega \varepsilon_{K L}-\frac{1}{\sqrt{\left(r^{2} B^{2}+\tilde{G}^{2}\right)}} \\
\times & \left(H_{1, \theta}+H_{1} \frac{C_{, \theta}}{C}\right)-\frac{H_{2}}{B}\left\{\frac{C^{\prime}}{C}-\frac{2(r B)(r B)^{\prime}+\tilde{G} \tilde{G}^{\prime}}{2\left(r^{2} B^{2}+\tilde{G}^{2}\right)}\right\} \\
= & -\frac{4 \pi}{\lambda+2 \xi R}\left\{\frac{1}{3} \rho \sigma_{I}+\frac{1}{B} q_{I}^{\prime}+\frac{q_{I I}}{\sqrt{r^{2} B^{2}+\tilde{G}^{2}}}\left(\frac{\tilde{G} \dot{B}}{B}+\frac{B_{, \theta}}{B}\right)\right\}
\end{aligned}
$$




$$
\begin{aligned}
& +\frac{\xi}{\lambda+2 \xi R} K^{\alpha} K^{\beta}\left\{\dot{R}\left(R_{\alpha \beta}-\frac{1}{3} R g_{\alpha \beta}\right)-\left(\nabla_{\alpha} R\right) R_{\beta \gamma} V^{\gamma}\right. \\
& \left.+\nabla_{\alpha}\left(\nabla_{\gamma} \nabla_{\beta} R\right) V^{\gamma}-\left(\nabla_{\alpha} \nabla_{\beta} R\right)^{\cdot}\right\} \text {, } \\
& \frac{1}{3} \dot{\varepsilon}_{I I}+\frac{1}{9} \varepsilon_{I I}\left(3 \Theta+\sigma_{I}-\sigma_{I I}\right)+\frac{1}{9} \varepsilon_{I}\left(2 \sigma_{I}+\sigma_{I I}\right)+\Omega \varepsilon_{K L}+\frac{1}{B}\left(H_{2}^{\prime}+H_{2} \frac{C^{\prime}}{C}\right) \\
& +\frac{H_{1}}{\sqrt{\left(r^{2} B^{2}+\tilde{G}^{2}\right)}}\left\{\frac{C_{, \theta}}{C}-\frac{B_{, \theta}}{B}-\tilde{G}\left(\frac{\dot{B}}{B}-\frac{\dot{C}}{C}\right)\right\}=-\frac{4 \pi}{\lambda+2 \xi R} \\
& \times\left\{\frac{1}{3} \rho \sigma_{I I}+\frac{1}{2 B} q_{I} \frac{\left(r^{2} B^{2}+\tilde{G}^{2}\right)^{\prime}}{r^{2} B^{2}+\tilde{G}^{2}}-\frac{\left(\tilde{G} \dot{q}_{I I}+q_{I I, \theta}\right)}{\sqrt{r^{2} B^{2}+\tilde{G}^{2}}}\right\} \\
& +\frac{\xi}{\lambda+2 \xi R} L^{\alpha} L^{\beta}\left\{\dot{R}\left(R_{\alpha \beta}-\frac{1}{3} R g_{\alpha \beta}\right)-\left(\nabla_{\alpha} R\right) R_{\beta \gamma} V^{\gamma}\right. \\
& \left.+\nabla_{\alpha}\left(\nabla_{\gamma} \nabla_{\beta} R\right) V^{\gamma}-\left(\nabla_{\alpha} \nabla_{\beta} R\right)^{\cdot}\right\} \\
& -\frac{1}{3}\left(\varepsilon_{I}+\varepsilon_{I I}\right)^{\cdot}-\frac{1}{3}\left(\varepsilon_{I}+\varepsilon_{I I}\right) \Theta-\frac{1}{9} \varepsilon_{I}\left(2 \sigma_{I I}+\sigma_{I}\right)-\frac{1}{9} \varepsilon_{I I}\left(2 \sigma_{I}+\sigma_{I I}\right) \\
& +\frac{1}{\sqrt{r^{2} B^{2}+\tilde{G}^{2}}}\left(H_{1, \theta}+H_{1} \frac{B_{, \theta}}{B}\right)-\frac{1}{B}\left\{H_{2}^{\prime}+H_{2} \frac{\left(r^{2} B^{2}+\tilde{G}^{2}\right)^{\prime}}{2\left(r^{2} B^{2}+\tilde{G}^{2}\right)}\right\} \\
& =\frac{4 \pi}{\lambda+2 \xi R}\left\{\frac{1}{3} \rho\left(\sigma_{I}+\sigma_{I I}\right)-\frac{1}{B} q_{I} \frac{C^{\prime}}{C}-\frac{q_{I I}}{\sqrt{r^{2} B^{2}+\tilde{G}^{2}}}\left(\frac{\tilde{G} \dot{C}}{C}+\frac{C_{, \theta}}{C}\right)\right\} \\
& +\frac{\xi}{\lambda+2 \xi R} S^{\alpha} S^{\beta}\left\{\dot{R}\left(R_{\alpha \beta}-\frac{1}{3} R g_{\alpha \beta}\right)-\left(\nabla_{\alpha} R\right) R_{\beta \gamma} V^{\gamma}\right. \\
& \left.+\nabla_{\alpha}\left(\nabla_{\gamma} \nabla_{\beta} R\right) V^{\gamma}-\left(\nabla_{\alpha} \nabla_{\beta} R\right)^{\cdot}\right\} \text {, } \\
& 2 \dot{\varepsilon}_{K L}+\varepsilon_{K L}\left(2 \Theta-\sigma_{I}-\sigma_{I I}\right)+\frac{\Omega}{3}\left(\varepsilon_{I}-\varepsilon_{I I}\right)+\frac{1}{B}\left[H_{1}^{\prime}+H_{1}\right. \\
& \left.\times\left\{\frac{2 C^{\prime}}{C}-\frac{\left(2(B r)(B r)^{\prime}+\tilde{G} \tilde{G}^{\prime}\right)}{2\left(r^{2} B^{2}+\tilde{G}^{2}\right)}\right\}\right]-\frac{1}{\sqrt{r^{2} B^{2}+\tilde{G}^{2}}} \times\left[H_{2, \theta}+H_{2}\right. \\
& \left.\times\left\{\frac{2 C_{, \theta}}{C}-\frac{2 B_{, \theta}}{B}-\tilde{G}\left(\frac{\dot{B}}{B}-\frac{\dot{C}}{C}\right)\right\}\right]=\frac{2 \pi}{(\lambda+2 \xi R) \sqrt{r^{2} B^{2}+\tilde{G}^{2}}} \\
& \times\left\{q_{I}\left(\tilde{G} \frac{\dot{B}}{B}+\frac{B, \theta}{B}\right)-\tilde{G} \dot{q}_{I}-q_{I, \theta}\right\}+\frac{2 \pi}{(\lambda+2 \xi R) B}\left\{-q_{I I}^{\prime}+q_{I I} \frac{\left(r^{2} B^{2}+\tilde{G}^{2}\right)^{\prime}}{2\left(r^{2} B^{2}+\tilde{G}^{2}\right)}\right\}
\end{aligned}
$$




$$
\begin{aligned}
& +\frac{\xi}{\lambda+2 \xi R} K^{\alpha} L^{\beta}\left\{\dot{R}\left(R_{\alpha \beta}-\frac{1}{3} R g_{\alpha \beta}\right)-\left(\nabla_{\alpha} R\right) R_{\beta \gamma} V^{\gamma}\right. \\
& \left.+\nabla_{\alpha}\left(\nabla_{\gamma} \nabla_{\beta} R\right) V^{\gamma}-\left(\nabla_{\alpha} \nabla_{\beta} R\right)^{\cdot}\right\}
\end{aligned}
$$

Addition of the above three equations gives

$$
\begin{aligned}
& \frac{\tilde{G}}{\sqrt{r^{2} B^{2}+\tilde{G}^{2}}}\left\{\frac{H_{2} \tilde{G}^{\prime}}{2 B \sqrt{r^{2} B^{2}+\tilde{G}^{2}}}+H_{1}\left(\frac{\dot{B}}{B}-\frac{\dot{C}}{C}\right)\right\} \\
= & \frac{4 \pi}{B(\lambda+2 \xi R)}\left\{q_{I}^{\prime}+q_{I}\left(\frac{C^{\prime}}{C}+\frac{\left(r^{2} B^{2}+\tilde{G}^{2}\right)^{\prime}}{2\left(r^{2} B^{2}+\tilde{G}^{2}\right)}\right)\right\} \\
+ & \frac{4 \pi}{\sqrt{r^{2} B^{2}+\tilde{G}^{2}}(\lambda+2 \xi R)}\left[q_{I I}\left\{\tilde{G}\left(\frac{\dot{B}}{B}+\frac{\dot{C}}{C}\right)+\frac{C_{, \theta}}{C}+\frac{B_{, \theta}}{B}\right\}+\tilde{G}_{\dot{q}_{I I}}+q_{I I, \theta}\right] \\
+ & \frac{\xi}{\lambda+2 \xi R}\left(K^{\alpha} K^{\beta}+L^{\alpha} L^{\beta}+S^{\alpha} S^{\beta}\right)\left\{\dot{R}\left(R_{\alpha \beta}-\frac{1}{3} R g_{\alpha \beta}\right)-\left(\nabla_{\alpha} R\right) R_{\beta \gamma} V^{\gamma}\right. \\
+ & \left.\nabla_{\alpha}\left(\nabla_{\gamma} \nabla_{\beta} R\right) V^{\gamma}-\left(\nabla_{\alpha} \nabla_{\beta} R\right)^{\cdot}\right\} .
\end{aligned}
$$

The transport equation is

$$
\tau h_{\mu}^{\nu} q_{\nu ; \alpha}^{t o t} V^{\alpha}+q_{\mu}^{t o t}=\kappa h_{\mu}^{\nu} T_{, \nu}+\frac{1}{2} \kappa T^{2}\left(\frac{\tau V^{\alpha}}{\kappa T^{2}}\right)_{; \alpha} q_{\mu}^{t o t} .
$$

A combination of conservation equation and transport equation gives

$$
\begin{aligned}
& -\tau\left(\frac{4}{3} \Theta h_{\mu \nu}+\sigma_{\mu \nu}+\Omega_{\mu \nu}\right) q^{\nu}-\frac{\tau}{8 \pi}\left[\left\{\nabla^{\nu} \nabla_{\mu}(\lambda+2 \xi R)-2 \xi R\right\}_{; \nu}\right. \\
& \left.-\delta_{\mu}^{\nu}\left\{2 \xi(\square R)_{; \nu}+\frac{1}{2}\left(\xi R^{2}\right)_{; \nu}\right\}\right]+\tau h_{\mu}^{\nu} q_{\nu ; \alpha}^{(D)} V^{\alpha}+q_{\mu}^{(D)}+\kappa h_{\mu}^{\nu} T_{, \nu} \\
& +\frac{1}{2} \kappa T^{2}\left(\frac{\tau V^{\alpha}}{\kappa T^{2}}\right)_{; \alpha} q_{\mu}^{t o t} .
\end{aligned}
$$

Following are the terms used in the paper

$$
\begin{aligned}
D_{1} & =\left[\frac{8 \pi \rho q_{I}^{t o t}}{3(\lambda+2 \xi R)}-\frac{\xi \nabla_{t} \nabla_{r} R}{B(\lambda+2 \xi R)}\right]\left[\frac{12 \pi \rho}{(\lambda+2 \xi R)}-\frac{2 R^{2} \xi}{(\lambda+2 \xi R)}+\frac{\xi}{(\lambda+2 \xi R)}\right. \\
& \left.\times\left\{2\left(g^{\delta \gamma} \nabla_{\delta} \nabla_{\gamma} R-V^{\delta} V^{\gamma} \nabla_{\delta} \nabla_{\gamma} R\right)+\epsilon^{\nu \mu \gamma} \epsilon_{\gamma \nu}^{\delta} \nabla_{\delta} \nabla_{\mu} R\right\}\right] \\
D_{2} & =\left[\frac{8 \pi \rho q_{I I}^{t o t}}{3(\lambda+2 \xi R)}-\frac{\xi \nabla_{t} \nabla_{\theta} R}{\sqrt{\tilde{G}^{2}+r^{2} B^{2}}(\lambda+2 \xi R)}\right]\left[\frac{12 \pi \rho}{(\lambda+2 \xi R)}-\frac{2 R^{2} \xi}{(\lambda+2 \xi R)}\right.
\end{aligned}
$$




$$
\begin{aligned}
& \left.+\frac{\xi}{(\lambda+2 \xi R)}\left\{2\left(g^{\delta \gamma} \nabla_{\delta} \nabla_{\gamma} R-V^{\delta} V^{\gamma} \nabla_{\delta} \nabla_{\gamma} R\right)+\epsilon^{\nu \mu \gamma} \epsilon_{\gamma \nu}^{\delta} \nabla_{\delta} \nabla_{\mu} R\right\}\right] \\
D_{3} & =-\frac{1}{8 \pi B}\left[\left\{\nabla^{\nu} \nabla_{1}(\lambda+2 \xi R)-2 \xi R R_{2}^{\nu}\right\}_{; \nu}-\delta_{1}^{\nu}\left\{2 \xi(\square R)_{; \nu}+\frac{1}{2}\left(\xi R^{2}\right)_{; \nu}\right\}\right] \\
& +\frac{T_{01}^{(D)}}{8 \pi}\left\{\frac{1}{\tau}+\frac{1}{2} D_{t}\left[\ln \left(\frac{\tau}{\kappa T^{2}}\right)\right]\right\}-\frac{1}{8 \pi B}\left(B T_{01}^{(D)}\right)_{; 0}, \\
D_{4} & =-\frac{1}{8 \pi \sqrt{r^{2} B^{2}+\tilde{G}^{2}}}\left[\left\{\nabla^{\nu} \nabla_{2}(\lambda+2 \xi R)-2 \xi R R_{2}^{\nu}\right\}_{; \nu}-\delta_{2}^{\nu}\left\{2 \xi(\square R)_{; \nu}\right.\right. \\
& \left.\left.+\frac{1}{2}\left(\xi R^{2}\right)_{; \nu}\right\}\right]+\frac{\left(\tilde{G} T_{00}^{(D)}+T^{(D)}\right)_{02}}{8 \pi}\left\{\frac{1}{\tau}+\frac{1}{2} D_{t}\left[\ln \left(\frac{\tau}{\kappa T^{2}}\right)\right]\right\} \\
& -\frac{1}{8 \pi \sqrt{r^{2} B^{2}+\tilde{G}^{2}}}\left(\tilde{G} T_{00}^{(D)}+T^{(D)}\right)_{; 0} \cdot \\
D_{5} & =\frac{\xi}{(\lambda+2 \xi R)}\left(K^{\alpha} K^{\beta}-\frac{1}{3} h_{\mu \nu} K^{\mu} K^{\nu} h^{\alpha \beta}\right) \nabla_{\alpha} \nabla_{\beta} R, \\
D_{6} & =\frac{\xi}{(\lambda+2 \xi R)}\left(L^{\alpha} L^{\beta}-\frac{1}{3} h_{\mu \nu} L^{\mu} L^{\nu} h^{\alpha \beta}\right) \nabla_{\alpha} \nabla_{\beta} R . \\
D_{7} & =\frac{\xi}{(\lambda+2 \xi R)}\left(L^{\alpha} L^{\beta}-\frac{1}{3} h_{\mu \nu} L^{\mu} L^{\nu} h^{\alpha \beta}\right) \nabla_{\alpha} \nabla_{\beta} R, \\
D_{8} & =\frac{\xi}{(\lambda+2 \xi R)} K^{\alpha} K^{\beta}\left\{\dot{R}\left(R_{\alpha \beta}-\frac{1}{3} R g_{\alpha \beta}\right)-\left(\nabla_{\alpha} R\right) R_{\beta \gamma} V^{\gamma}\right. \\
& \left.+\nabla_{\alpha}\left(\nabla_{\gamma} \nabla_{\beta} R\right) V^{\gamma}-\left(\nabla_{\alpha} \nabla_{\beta} R\right)^{\cdot}\right\}, \\
D_{9} & =\frac{\xi}{(\lambda+2 \xi R)} L^{\alpha} L^{\beta}\left\{\dot{R}\left(R_{\alpha \beta}-\frac{1}{3} R g_{\alpha \beta}\right)-\left(\nabla_{\alpha} R\right) R_{\beta \gamma} V^{\gamma}\right. \\
& \left.+\nabla_{\alpha}\left(\nabla_{\gamma} \nabla_{\beta} R\right) V^{\gamma}-\left(\nabla_{\alpha} \nabla_{\beta} R\right)^{\cdot}\right\} .
\end{aligned}
$$

\section{References}

[1] Abbott, B.P. et al: Phys. Rev. Lett. 116(2016)061102.

[2] Hawking, S.W.: Phys. Rev. Lett. 26(1971)1344.

[3] Wagoner, R.V.: Astrophys. J. 278(1984)345.

[4] Flanagan, E.E. and Hughes, S.A.: New J. Phys. 7(2005)204. 
[5] Ellis, G.F.R.: J. Math. Phys. 8(1967)1171.

[6] Lemos, J.P.S.: Phys. Rev. Lett. 68(1992)1447.

[7] Herrera, L.: Int. J. Mod. Phys. D 20(2011)1689; Sharif, M. and Yousaf, Z.: Astrophys. Space Sci.: 352(2014)321; Yousaf, Z., Bamba, K. and Bhatti, M.Z.: Phys. Rev. D 93(2016)124048.

[8] Herrera, L., Di Prisco, A., Ospino, J. and Carot, J.: Phys. Rev. D 91(2015)124015.

[9] Hawking, S.W. and Ellis, G.F.R.: The Large Scale Structure of Spacetime (Cambridge University Press, 1973).

[10] Hernandez Jr, W.C.: Phys. Rev. 153(1967)1359.

[11] Herrera, L., Di Prisco, A. and Ospino, J.: Phys. Rev. D 91(2015)024010.

[12] Newman, E.T. and Penrose, R.: J. Math. Phys. 3(1962)566; Hawking, S.W.: Astrophys. J. 145(1966)544.

[13] Matte, A.: Can. J. Math. 5(1953)1.

[14] Bertschinger, E. and Hamilton, A.J.S.: Astrophys. J. 435(1994)1.

[15] Maartens, R. and Basset, B.A.: Class. Quantum Grav. 15(1998)705.

[16] Ferrando, J.J. and Sáez, J.A.: Class. Quantum Grav. 29(2012)075012.

[17] Starobinsky, A.A.: Phys. Lett. B 91(1980)99.

[18] Bamba, K., Geng, C.Q. and Lee, C.C.: J. Cosmol. Astropart. Phys. 08(2010)021.

[19] Hu, W. and Sawicki, I.: Phys. Rev. D 76(2007)064004.

[20] Tsujikawa, S.: Phys. Rev. D 77(2008)023507.

[21] Cembranos, J.A.R., Cruz-Dombriz, A.D.L. and Núez, B.M.: J. Cosmol. Astropart. Phys. 04(2012)021.

[22] Sharif, M. and Yousaf, Z.: Phys. Rev. D 88(2013)024020; Eur. Phys. J. C 73(2013)2633; Astropart. Phys. 56(2014)19; Mon. Not. R. Astron. Soc. 440(2014)3479. 
[23] Sharif, M. and Nasir, Z.: Gen. Relativ. Gravit. 47(2015)85.

[24] Sharif, M. and Nasir, Z.: Commun. Theor. Phys. 64(2015)139.

[25] Capozziello, S., De Laurentis, M. and Stabile, A.: Class. Quantum Grav. $\mathbf{2 7}(2010) 165008$.

[26] Rippl, S., Elst, H.V., Tavakol, R. and Taylor, D.: Gen. Relativ. Gravit. 28(1995)193.

[27] Näf, J. and Jetzer, P.: Phys. Rev. D 84(2011)024027.

[28] Corda, C.: Int. J. Mod. Phys. A 23(2008)1521; Yang, L., Lee, C. and Geng, C.: J. Cosmol. Astropart. Phys. 08(2011)29.

[29] Carloni, S., Ananda, K.N., Dunsby, P.K.S. and Abdelwahab, M.E.S.: arXiv:0812.2211 [astro-ph].

[30] Carlson, G.T., Jr. and Safko, J.L.: Ann. Phys. 128(1980)131.

[31] Stephani, H., Kramer, D., MacCallum, M., Honselaers, C. and Herlt, E.: Exact Solutions of Einstein's Field Equations (Cambridge University Press, 2003).

[32] Herrera, L., Carot, J. and Di Prisco, A.: Phys. Rev. D 76(2007)044012.

[33] Bel, L.: Ann. Inst. H Poincaré 17(1961)37.

[34] Garcia-Parrado Gomez Lobo, A.: Class. Quantum Grav. 25(2008)015006.

[35] De Felice, A. and Tsujikawa, S.: Living Rev. Relativ. 13(2010)3; Sotirou, T.P. and Faraoni, V.: Rev. Mod. Phys. 82(2010)451.

[36] Cembranos, J.A.R.: Phys. Rev. Lett. 102(2009)141301; J. Phys. Conf. Ser. 315(2011)012004. 